\title{
Equilibrium properties of short field-reversed configurations
}

Ross L. Spencer

ross_spencer@byu.edu

M. Tuszewski

Follow this and additional works at: https://scholarsarchive.byu.edu/facpub

Part of the Astrophysics and Astronomy Commons, and the Physics Commons

\section{Original Publication Citation}

Tuszewski, M. and R. L. Spencer."Equilibrium properties of short field-reversed configurations."

Physics of Fluids 29 (1986): 3711-3714.

\section{BYU ScholarsArchive Citation}

Spencer, Ross L. and Tuszewski, M., "Equilibrium properties of short field-reversed configurations" (1986). Faculty Publications. 749.

https://scholarsarchive.byu.edu/facpub/749

This Peer-Reviewed Article is brought to you for free and open access by BYU ScholarsArchive. It has been accepted for inclusion in Faculty Publications by an authorized administrator of BYU ScholarsArchive. For more information, please contact ellen_amatangelo@byu.edu. 


\title{
Equilibrium properties of short field-reversed configurations
}

\author{
M. Tuszewski \\ Los Alamos National Laboratory, Los Alamos, New Mexico 87545 \\ R. L. Spencer \\ Brigham Young University, Provo, Utah 84602
}

(Received 28 January 1986; accepted 12 August 1986)

Some features of short field-reversed configuration (FRC) equilibria relevant to transport and stability are studied numerically and analytically. It is shown that magnetic field curvature effects significantly increase the FRC magnetization for plasma elongations $\epsilon \leqslant 4$.

\section{INTRODUCTION}

A field-reversed configuration (FRC) is an elongated compact toroid without a toroidal field. ${ }^{1}$ These plasmas are formed in prolate theta pinches with a reversed bias field. In most experiments, FRC's have aspect ratios $\epsilon$ (the ratio of the separatrix length to the diameter) sufficiently large so that magnetic field curvature is negligible over the central portion of the separatrix. For such cases, assuming no plasma pressure outside the separatrix and an infinitely long flux conserver of uniform radius, an axial equilibrium constraint (first derived by Barnes) is obtained ${ }^{1}$ :

$$
\langle\beta\rangle=\frac{1}{r_{s}^{2}} \int_{0}^{r_{s}} \frac{p}{p_{M}} 2 r d r=1-\frac{1}{2} \frac{x_{s}^{2}}{2},
$$

where $\beta=p / p_{M}$ is the plasma pressure normalized to the maximum pressure at the field null and where $x_{s}=r_{s} / r_{w}$ is the ratio of midplane separatrix radius to coil radius. The average beta condition of Eq. (1) is a stringent constraint on the FRC radial pressure profile. It has been found to be consistent with the available experimental data, ${ }^{2}$ and has often been used in equilibrium and transport studies. Various corrections to Eq. (1) have been derived to account for finite particle orbits, ${ }^{3}$ plasma pressure on open field lines, ${ }^{4}$ end mirrors, ${ }^{4,5}$ and toroidal field. ${ }^{4}$

Magnetic field curvature may significantly modify Eq. (1) if FRC equilibria are produced with sufficiently small values of $\epsilon$. Such small values of $\epsilon$ could result from separatrix length shrinkage, ${ }^{6}$ which occurs whenever energy losses exceed poloidal flux loss. Such short equilibria are currently being studied experimentally. In the VLX device ${ }^{7}$ FRC confinement in a coil of aspect ratio (ratio of uniform coil region to coil diameter) variable in the range $1-4$ is being investigated, and in the FRX-C device ${ }^{8}$ short FRC equilibria with $\epsilon$ in the range 3-4 have been recently obtained with a coil aspect ratio of 2.6. Even shorter FRC equilibria may be obtained in a modified version of this device ${ }^{9}$ with a coil aspect ratio of about 2.

\section{EQUILIBRIUM COMPUTATIONS}

In this paper, we compute the effect of field curvature on FRC equilibria. Short equilibria were obtained in straight cylinder geometry (no end mirrors) by using the same code as in previous work. ${ }^{4}$ In each of the cases discussed in this paper, the equilibrium length was adjusted by changing the total toroidal current; $\beta_{s}$ (the pressure at the separatrix normalized to the pressure at the field null) and $x_{s}$ were held fixed by adjusting the pressure profile, $p(\psi)$. For each equilibrium, the integral given in Eq. (1) was computed and the ratio $\langle\beta\rangle /\left(1-\frac{1}{2} x_{s}^{2}\right)$ was obtained. These ratios are shown as functions of $\epsilon$ in Fig. 1 for various values of $x_{s}$ and $\beta_{s}$. The open circles correspond to $x_{s}=0.4$ and $\beta_{s}=0.6$ and the solid circles correspond to $x_{s}=0.6$ and $\beta_{s}=0.3$. Also shown with a triangle in Fig. 1 is the analytical spherical Hill's vortex equilibrium $\left(\beta_{s}=0\right)$ which is a solution in a uniform flux conserver ${ }^{10}$ in the limit of $x_{s} \rightarrow 0$. For this case, one has $\langle\beta\rangle=\frac{2}{3}$ at $\epsilon=1$. The equilibria represented in Fig. 1 by open circles do not appear to map towards the Hill's vortex value, presumably because of the finite values of $\boldsymbol{x}_{s}$ and $\beta_{s}$. Fixing $\beta_{s}$ and reducing $\epsilon$ produced rather odd-looking equilibria with a lot of magnetofluid outside the separatrix. To see what happens when this fluid is removed, two additional equilibria (open circles with a bar) were computed with $x_{s}=0.4$ and $\beta_{s}=0.1$. These equilibria have lower values of $\langle\beta\rangle$ than those with $x_{s}=0.4$ and $\beta_{s}=0.6$ and better extrapolate toward the Hill's vortex value. The results

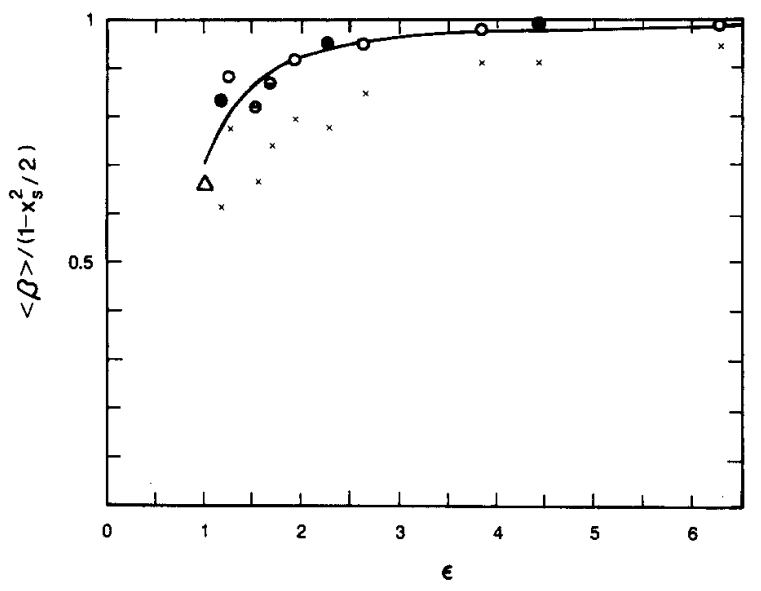

FIG. 1. Computed values of $\langle\beta\rangle$ relative to elongated values as functions of $\epsilon$. The open circles are for $x_{s}=0.4$ and $\beta_{s}=0.6$, the open circles with a bar are for $x_{s}=0.4$ and $\beta_{s}=0.1$, and the solid circles are for $x_{s}=0.6$ and $\beta_{s}=0.3$. The triangle is for the Hill's vortex case with $x_{s}=\beta_{s}=0$, and the solid line is the analytical expression of Eq. (3) with $x_{s}=0.5$. For each case, the value $\langle\beta\rangle_{v}$, the average of beta over the separatrix volume, normalized to $1-\frac{1}{2} x_{s}^{2}$, is shown with a cross. 
in Fig. 1 show that curvature effects are negligible for $\epsilon \geqslant 4$ but rapidly become significant as $\epsilon$ approaches unity. Comparison between the various cases suggests that curvature effects are somewhat reduced by larger values of $x_{s}$ and $\beta_{s}$.

For highly elongated FRC's, it is normally assumed that $\langle\beta\rangle$, the midplane-averaged beta, is the same as $\langle\beta\rangle_{v}$, the volume-averaged beta. Because $\langle\beta\rangle$ is easily determined experimentally, it is often used, for the case of highly elongated FRC's, as a substitute for the more physical quantity $\langle\beta\rangle_{\nu}$. However, as $\epsilon$ approaches 1 , the values of $\langle\beta\rangle_{v}$ can diverge significantly from the values of $\langle\beta\rangle$. This is illustrated in Fig. 1 where the values of $\langle\beta\rangle_{v}$, normalized to $1-\frac{1}{2} x_{s}^{2}$, are shown with crosses for each computed case. Note that both $\langle\beta\rangle$ and $\langle\beta\rangle$, decrease with decreasing $\epsilon$. However, the ratio $\langle\beta\rangle_{v} /\langle\beta\rangle$ decreases from 0.95 to 0.69 as $\epsilon$ decreases from 6 to 1 . Hence, there is no obvious relation between $\langle\beta\rangle$ and $\langle\beta\rangle_{v}$; furthermore, because of possible wide variations in the axial distribution of flux surfaces with variations in $p(\psi),{ }^{4}$ a simple relation between $\langle\beta\rangle$ and $\langle\beta\rangle_{v}$ may be quite difficult to find.

\section{ANALYTIC MODEL}

In order to gain more insight, an attempt was made to fit a simple analytical model for curvature effects. The numerical equilibria revealed midplane radial profiles of total pressure $\left(p+B^{2} / 2 \mu_{0}\right)$ with a drop largely localized in the vicinity of the separatrix. Three examples are given in Fig. 2 for various values of $\epsilon$ and for $x_{s}=0.4$. These examples suggest an approximate model with uniform regions of total pressure inside and outside the separatrix. An example for curve (c) is sketched with a dotted line on Fig. 2. We assume $p+B^{2}$ / $2 \mu_{0}=p_{M}$ inside the separatrix and $p+B^{2} / 2 \mu_{0}=p_{M}(1-\delta)$ outside the separatrix. Note that the discontinuity in total pressure in this model implies that $\mathbf{B} \cdot \mathbf{\nabla B}$ is proportional to a delta function at the separatrix, i.e., implies that this quanti-

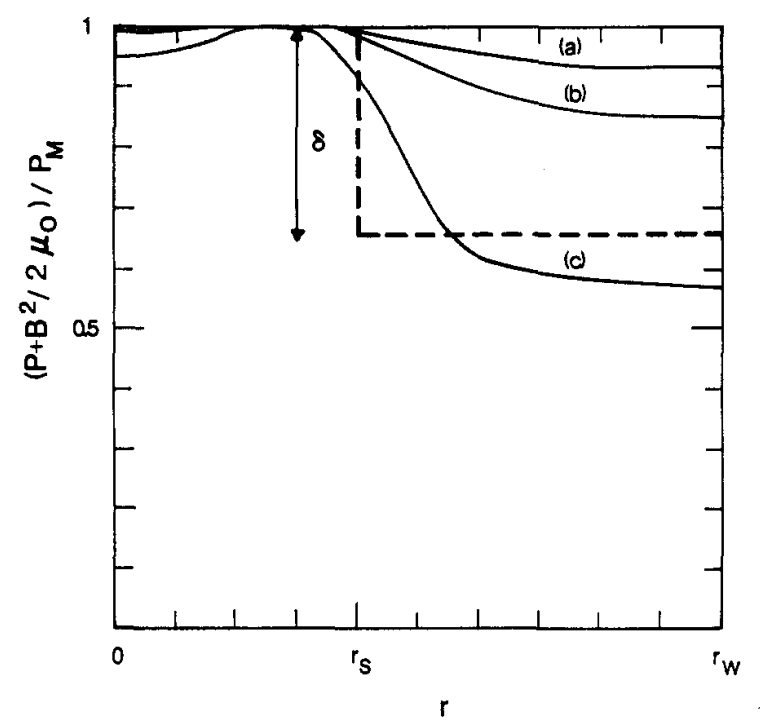

FIG. 2. Computed radial pressure profiles relative to the maximum pressure $p_{M}$ for $x_{s}=0.4$ and (a) $\epsilon=3.9$, (b) $\epsilon=2.7$, and (c) $\epsilon=1.4$. The dotted line is an approximate sharp-boundary model for curve (c). ty is quite large and localized near the separatrix. This is a rather drastic approximation, but since the quantity of interest, $\langle\beta\rangle$, is an integrated quantity, it is not as bad as might be expected. Note that as $\epsilon$ becomes large, this model is trivially exact with $\delta=0$. With this model the integral for $\langle\beta\rangle$ in Eq. (1) can be evaluated, following the derivation outlined in previous work. ${ }^{1}$

We start with Eq. (A7) of Ref. 1. When normalized to $\pi r_{s}^{2} p_{M}$, this equation becomes

$$
\begin{aligned}
\langle\beta\rangle= & \left.\frac{1}{\pi r_{s}^{2}} \int_{0}^{r_{w}} \frac{B^{2}}{2 \mu_{0} p_{M}} 2 \pi r d r\right|_{z=0} \\
& -\left.\frac{1}{\pi r_{s}^{2}} \int_{0}^{r_{w}} \frac{B^{2}}{2 \mu_{0} p_{M}} 2 \pi r d r\right|_{z>0} .
\end{aligned}
$$

The first integral on the right-hand side of Eq. (2) can be evaluated using $B^{2} / 2 \mu_{0} p_{M}=1-p / p_{M}$ for $r<r_{s}$ and $B^{2} /$ $1 \mu_{0} p_{M}=1-\delta(p=0)$ for $r>r_{s}$. In the second integral we use flux conservation, $\left.B\right|_{z>0}=B_{w}\left(1-x_{s}^{2}\right)$, and our modified radial pressure balance, $B_{w}^{2} / 2 \mu_{0} p_{M}=1-\delta$. With these substitutions, and some rearrangement, Eq. (2) can be written

$$
\langle\beta\rangle=1-x_{s}^{2} / 2-(\delta / 2)\left(1-x_{s}^{2}\right) .
$$

We now make an estimate of the quantity $\delta$. With field-line curvature included, radial pressure balance, in the midplane, becomes

$$
p+\frac{B^{2}}{2 \mu_{0}}=\int \frac{B^{2}}{\mu_{0} \rho} d r
$$

where $\rho$ is the radius of curvature of the magnetic field lines. To a good approximation, FRC separatrices $r_{s}(z)$ have been shown experimentally ${ }^{2,4}$ to be elliptical in shape, so that at $r=r_{s}$ and $z=0$ one obtains $\rho=-r_{s} \epsilon^{2}$. Assuming that the integrated curvature factor $\delta$ is proportional to $\epsilon^{-2}$, we write $\delta=\alpha / \epsilon^{2}$. The coefficient $\alpha$ can now be estimated by computing it for the simplest finite-length FRC equilibrium, the Hill's vortex. We do so by requiring Eq. (3) to hold for this equilibrium. With $\langle\beta\rangle=2 / 3, x_{s} \ll 1$, and $\epsilon=1$, we obtain $\delta=\alpha=2 / 3$. We therefore assume $\delta=2 / 3 \epsilon^{2}$ and rewrite Eq. (3) as

$$
\langle\beta\rangle=1-x_{s}^{2} / 2-\left(1-x_{s}^{2}\right) / 3 \epsilon^{2} .
$$

The solid line on Fig. 1 corresponds to Eq. (4) with $x_{s}=0.5$. This curve fits well the numerical data over the entire range of $\epsilon$. Equation (4) provides a simple approximation to $\langle\beta\rangle$ for arbitrary aspect ratio $\epsilon$ that can easily be evaluated experimentally. The factor $1-x_{s}^{2}$ that appears in Eq. (4) implies decreased curvature effects with larger values of $x_{s}$, in qualitative agreement with the numerical data of Fig. 1 . This can probably be attributed to the effect of the flux conserving wall; at large $x_{s}$, the plasma is pushed against the straight wall, and tends to become flat. The numerical data of Fig. 1 also suggest that curvature effects are lessened with increasing $\beta_{s}$; this effect is not included in Eq. (4).

The FRC total temperature $T$ is commonly inferred from radial pressure balance once $B_{W}$ at radius $r=r_{W}$ and $n_{M}$ at radius $r=r_{s} / 2^{1 / 2}$ are determined from excluded flux measurements and from side-on interferometry. Therefore, the expressions $p_{M}=n_{M} T=B_{w}^{2} / 2 \mu_{0}(1-\delta)$ and $\delta=2 /$ 
$3 \epsilon^{2}$ provide a temperature estimate for cases with low values of $\epsilon$.

The values of $\langle\beta\rangle$ given by Eq. (4) can be used to extend existing one-dimensional transport models, such as the one of Ref. 3, to the range $\epsilon \sim 2-4$. In this range, end effects that reduce ${ }^{11,12}$ confinement times by a factor $\epsilon^{2} /\left(\epsilon^{2}+1\right)$ remain small while the decreased $\langle\beta\rangle$ (and therefore decreased pressure gradients) should yield improved cross-field transport.

$$
\begin{aligned}
& \text { A parameter of importance for FRC stability is } \\
& \bar{s}=\int_{R}^{r_{s}} \frac{r d r}{r_{s} \rho_{i}},
\end{aligned}
$$

the approximate number ${ }^{13}$ of ion gyroradii, $\rho_{i}$, between the field null $R$ and the separatrix $r_{s}$. A recent kinetic theory ${ }^{14}$ of the internal tilt mode suggests sufficiently small growth rates for present experiments with $\vec{s}<1-2$ to be stable, but observable instability in future experiments with $\bar{s}>3-4$. If $\bar{s}$ has the same significance with regard to stability for these short equilibria, then such equilibria might represent a simpler way to study the tilt mode. Their shorter length, however, could substantially alter the eigenfunction (for instance, by changing it from an axial displacement to a rigid rotation, or by changing the important instability from an internal tilt mode to an external tilt mode, or by changing it into some combination of all of these) making it difficult to compare with the physics of long equilibria. For cases with small values of $\epsilon$, the usual one-dimensional estimates ${ }^{12}$ of $\bar{s}$ become inaccurate. Since $\bar{s}$ is proportional to $\phi / r_{s}$, where $\phi$ is the FRC poloidal flux, and where we have assumed constant ion temperature, one can readily compute the relative increase of $\bar{s}$ over its elongated value, for a given value of $x_{s}$, by calculating $\phi$ ratios. The ratios $\bar{s} / \bar{s}_{\epsilon>1}=\phi / \phi_{\epsilon>1}$ from the numerical data of Fig. 1 are shown in Fig. 3. For the cases with $x_{s}=0.4$ we used an equilibrium with $\epsilon=6.3$ to obtain $\phi_{\epsilon>1}$, and for $x_{s}=0.6$ we used an equilibrium with $\epsilon=4.5$. We observe from Fig. 3 significant relative increases of $\bar{s}$ for $\epsilon<3$ that correspond to the enhanced magnetization suggested in Fig. 1 by lower values of $\langle\beta\rangle$. The reduction of curvature effects with larger values of $x_{s}$ and $\beta_{s}$ noted previously is also apparent in Fig. 3.

For elongated FRC's, Eq. (1) has been used ${ }^{1,6}$ to provide bounds on the FRC poloidal flux $\phi$. For short FRC's, Eq. (4) can be used in a similar way to obtain

$$
\begin{aligned}
\pi r_{w}^{2} B_{w} & \frac{x_{s}^{4}}{4}\left(1+\frac{\delta}{x_{s}^{2}(1-\delta)}\right) \\
& <\phi \leqslant \pi r_{w}^{2} B_{w} \frac{x_{s}^{3}}{2^{3 / 2}}\left(1+\frac{\delta}{x_{s}^{2}(1-\delta)}\right)^{1 / 2} .
\end{aligned}
$$

The bounds for $\phi$ given in Eq. (5) are higher than those of elongated FRC's (corresponding to $\delta=0$ ). It is often assumed $^{6}$ that the elongated FRC's of present experiments have values of $\phi$ much closer to the upper bound than to the lower bound. This is still true for the short numerical equilibria of this paper. Neglecting variations in $B_{w}$, the upper bound of Eq. (5) implies

$$
\frac{\phi}{\phi_{\epsilon>1}}=\frac{\left[1+\delta / x_{s}^{2}(1-\delta)\right]^{1 / 2}}{\left[1+\delta_{\epsilon>1} / x_{s}^{2}\left(1-\delta_{\epsilon>1}\right)\right]^{1 / 2}} .
$$

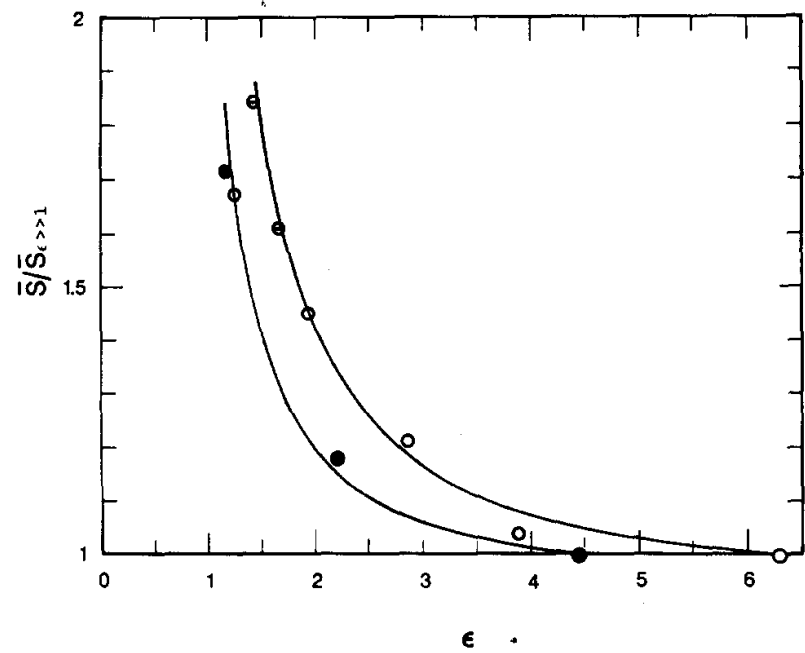

FIG. 3. Computed values of $\bar{s}$ for short equilibria, normalized to the corresponding values of $\bar{s}_{\epsilon>1}$ for highly elongated equilibria ( $\epsilon=6.3$ for $x_{s}=0.4$ and $\epsilon=4.5$ for $x_{s}=0.6$ ) are displayed as functions of $\epsilon$. The various cases are the same as those of Fig. 1. The solid lines correspond to the relation $\bar{s} \sim \phi \sim\left[1+\delta / x_{s}^{2}(1-\delta)\right]^{1 / 2}$, normalized to the same expression evaluated for $\epsilon=6.3$ ( $x_{s}=0.4$, upper curve) and for $\epsilon=4.5$ ( $x_{s}=0.6$, lower curve).

With $\delta=2 / 3 \epsilon^{2}$, this relation is shown with solid lines in Fig. 3 for $x_{s}=0.4$ and for $x_{s}=0.6$. The quantity $\delta_{\epsilon>1}$ is a value of $\delta$ corresponding to $\epsilon=6.3$ for $x_{s}=0.4$ and to $\epsilon=4.5$ for $x_{s}=0.6$. We observe from Fig. 3 that the numerical values of normalized $\bar{s}$ values are indeed in good agreement with the approximate upper-bound scaling. The relation

$$
\phi \sim B_{w} x_{s}^{3}\left[1+\delta / x_{s}^{2}(1-\delta)\right]^{1 / 2}
$$

may therefore be a useful way to use simple magnetic measurements in present experiments to estimate the flux confinement times of short FRC's. Similarly, the values of $\langle\beta\rangle_{0}$ shown in Fig. 1 could perhaps be used to estimate the energy confinement times of short FRC's from the relation $E \sim\langle\beta\rangle_{v} V B_{w}^{2} /(1-\delta)$, where $E$ is the plasma energy and $V$ is the separatrix volume.

\section{CONCLUSIONS}

In conclusion, some features of short FRC equilibria have been studied numerically. Specifically, it is shown that magnetic field curvature significantly increases the FRC magnetization for elongations $\epsilon \leqslant 4$. This should improve FRC transport and might decrease the effectiveness of kinetic stabilization. The transport parameter $\langle\beta\rangle$ is computed and a simple analytical approximation for it is derived. The relative increases of the stability parameter $\bar{s}$ and of the FRC trapped flux $\phi$ over one-dimensional estimates are evaluated. It is found that curvature effects are somewhat reduced by increased values of $x_{s}$ and $\beta_{s}$.

\section{ACKNOWLEDGMENT}

This research was supported by the United States Department of Energy. 
${ }^{1}$ W. T. Armstrong, R. K. Linford, J. Lipson, D. A. Platts, and E. G. Sherwood, Phys. Fluids 24, 2068 (1981).

${ }^{2}$ M. Tuszewski, Plasma Phys. 26, 991 (1984).

${ }^{3}$ M. Tuszewski and R. K. Linford, Phys. Fluids 25, 765 (1982).

${ }^{4}$ R. L. Spencer and M. Tuszewski, Phys. Fluids 28, 1810 (1985).

${ }^{5} K$. Suzuki and S. Hamada, J. Phys. Soc. Jpn. 53, 16 (1984).

${ }^{6} \mathrm{M}$. Tuszewski, W. T. Armstrong, R. R. Bartsch, R. E. Chrien, J. C. Cochrane, Jr., R. W. Kewish, Jr., P. Klingner, R. K. Linford, K. F. McKenna, D. J. Rej, E. G. Sherwood, and R. E. Siemon, Phys. Fluids 25, 1696 (1982)

7'J. A. Wilson, Z. A. Pietrzyk, and D. E. Lotz, Bull. Am. Phys. Soc. 29, 1360 (1984).

${ }^{8} \mathbf{M}$. Tuszewski, in Proceedings of the 7th Symposium on the Physics and Technology of Compact Toroids, Santa Fe, 1985 (Los Alamos National
Laboratory, Los Alamos, NM, 1986), p. 142.

${ }^{9}$ M. Tuszewski, R. E. Chrien, E. J. Yavornik, W. T. Armstrong, W. Hugrass, R. K. Linford, K. F. McKenna, D. J. Rej, and R. E. Siemon, in Proceedings of the 7th Symposium on the Physics and Technology of Compact Toroids, Santa Fe, 1985 (Los Alamos National Laboratory, Los Alamos, NM, 1986), p. 152.

${ }^{10}$ R. L. Spencer and D. W. Hewett, Phys. Fluids 25, 1365 (1982).

${ }^{11}$ S. P. Auerbach and W. C. Condit, Nucl. Fusion 21, 927 (1981).

${ }^{12}$ K. Nguyen and T. Kammash, Plasma Phys. 24, 177 (1982).

${ }^{13}$ J. T. Slough, A. L. Hoffman, R. D. Milroy, D. G. Harding, and L. C. Steinhauer, Nucl. Fusion 24, 1537 (1984).

${ }^{14}$ J. L. Schwarzmeier, H. R. Lewis, D. C. Barnes, and C. E. Seyler, Bull Am. Phys. Soc. 29, 1327 (1984). 